\title{
Lymphangiogenesis in kidney cancer: Expression of VEGF-C, VEGF-D and VEGFR-3 in clear cell and papillary renal cell carcinoma
}

\author{
S. BIERER ${ }^{1}$, E. HERRMANN ${ }^{1}$, T. KÖPKE ${ }^{1}$, J. NEUMANN ${ }^{2}$,

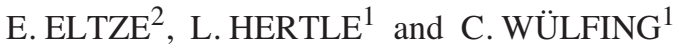 \\ ${ }^{1}$ Department of Urology; ${ }^{2}$ Gerhard Domagk-Institute of Pathology, \\ University Hospital Münster, 48149 Münster, Germany
}

Received March 28, 2008; Accepted May 22, 2008

DOI: 10.3892/or_00000065

\begin{abstract}
The vascular endothelial growth factors VEGF-C, VEGF-D and its receptor, VEGFR-3, are overexpressed in different malignancies and associated with lymph node metastasis and poor prognosis. We analysed these factors in clear cell (ccRCC) and papillary (pRCC) renal cell carcinoma (RCC). The results were correlated with various clinicopathological parameters (CPP). We constructed a tissue microarray with tumor samples of $135(81 \%)$ ccRCC and 31 (19\%) pRCC. After immunohistochemical staining using polyclonal antibodies for VEGF-C, VEGF-D and VEGFR-3, a semiquantitative analysis was performed to determine the levels of expression. The results were compared between the two subgroups and were correlated with CPP. In the two subgroups the expression of VEGF-C was significantly correlated with that of VEGF-D $(\mathrm{p}<0.001)$. There was an increased expression of VEGF-C in $11 \%$ of ccRCC and $36 \%$ of $\mathrm{pRCC}(\mathrm{p}=0.002)$. VEGF-D expression was positive by means of analysis in $22 \%$ of ccRCC and $42 \%$ of pRCC $(\mathrm{p}=0.039)$. There was no significant difference regarding the expression of VEGFR-3 between the subgroups (44\% ccRCC and $61 \% \mathrm{pRCC}, \mathrm{p}=0.11$ ). No correlation was found between the expression of the analysed parameters and CPP (TNM, grading, progression-free survival and overall survival) in either the entire group or in the two subgroups. In summary, ccRCC and pRCC show a different expression pattern of the analysed lymphangiogenic factors. Further studies are necessary to confirm these results and to determine whether the VEGF-C/VEGF-D/VEGFR-3-axis can play a role as a
\end{abstract}

Correspondence to: Dr Stefan Bierer, Department of Urology, University Hospital Münster, Albert Schweitzer-Strasse 33, 48149 Münster, Germany

E-mail: bierers@mednet.uni-muenster.de

Key words: renal cell carcinoma, lymphangiogenesis, immunohistochemistry, clear cell, papillary, vascular endothelial growth factor prognostic tool or a target for therapeutic intervention in renal cell carcinoma.

\section{Introduction}

Renal cell carcinoma (RCC) accounts for $\sim 3 \%$ of cancers in adults as well as $85 \%$ of all primary malignant kidney tumors (1). The 5-year survival rate for all stages of RCC has improved due to an important stage migration, whereby the majority of patients are currently diagnosed with localized disease (2). Over the past decades there has been a rising incidence in the United States not only of localized RCC but also of advanced stages, and the overall mortality of kidney cancer increased slightly during this period of time (1). Moreover, $\sim 25 \%$ of patients are still diagnosed with advanced or metastatic disease, which is associated with poor prognosis and outcome.

However, rapid advances and exciting new therapeutic strategies in the field of RCC have emerged in recent years. In particular, the discovery and analysis of molecular systems which control the formation of blood vessels have provided great insight into the potential of angiogenesis to influence tumor growth and spread in RCC. Thus, the vascular endothelial growth factor (VEGF) and platelet-derived growth factor (PDGF) signalling pathways have been identified as viable for anticancer therapy and the multitargeted receptor tyrosine kinase inhibitors sunitinib (3) and sorafenib (4) have demonstrated improved efficacy in the treatment of advanced and/or metastatic RCC. Recent evidence shows that temsirolimus, an inhibitor of the mammalian target of rapamycin (5), and bevacizumab, an anti-VEGF monoclonal antibody (6), provide benefits in the treatment of RCC.

This confirms the theory that inhibiting tumor-induced angiogenesis is an attractive strategy for therapeutic intervention in RCC.

In contrast, data focusing on lymphangiogenesis in RCC are scant which may be due to the fact that lymphatic metastases in RCC occur less frequently compared to haematogenous tumor dissemination. However, there is lymphatic tumor spread in RCC and patients with nodepositive tumors have a more aggressive disease than even the worst variant of localized disease (7). This possibly justifies 
more aggressive treatment in patients with lymph node metastasis, such as nephrectomy including lymphadenectomy and adjuvant systemic therapy (8). Therefore, understanding the molecular processes of lymphangiogenesis and lymphatic tumor spread in RCC is of special interest. This may help to improve existing prognostic models and possibly identify new targets for therapeutic intervention.

This study aimed to further evaluate lymphangiogenic parameters in clear cell RCC (ccRCC) and papillary RCC (pRCC). Since the VEGF-C/VEGF-D/VEGFR-3-axis appears to be the most validated signalling pathway for tumor-induced lymphangiogenesis and an attractive target for therapeutic intervention (9) we performed a semiquantitative analysis of the expression of these parameters after immunohistochemical staining. The results were correlated with various clinicopathological parameters (CPP).

\section{Materials and methods}

Patients. One hundred and sixty-six patients treated with nephrectomy for localized or advanced or metastatic RCC at our institution between 1991 and 2004 were selected from our RCC database: 135 (81\%) of the patients had ccRCC and $31(19 \%)$ pRCC, respectively. Histological slides and formalinfixed, paraffin-embedded tumor tissue blocks of the 166 patients were obtained from the Gerhard Domagk-Institute of Pathology (University Hospital Münster, Germany). The slides were reviewed and representative tumor tissue blocks were selected for constructing a tissue microarray (TMA). A database comprising of clinical and pathological data according to the TNM system was created (Table I). For each of the 166 samples a representative tumor block was selected as a donor block for TMA. Using a haematoxylin and eosin (H\&E)stained slide, two morphologically representative regions were defined for each of the samples. From these regions, cylindrical core tissue specimens (diameter $=0.6 \mathrm{~mm}$ ) were obtained and arrayed precisely into a new recipient paraffin block (20x35 mm) using a custom-built precision instrument (Beecher Instruments, Silver Spring, MD, USA). From the 332 tumor samples available, several tissue array blocks were prepared.

Immunohistochemistry. The paraffin-embedded tissue array blocks were cut into $3-\mu \mathrm{m}$ slices and mounted on poly-Llysine-coated glass slides. Tissue slides were dewaxed in xylene, rehydrated in a graded series of alcohol and rinsed in $0.01 \mathrm{M}$ tris buffer ( $\mathrm{pH} 7.3$ ). Immunohistochemical staining for VEGF-C, VEGF-D and VEGFR-3 was performed in a multistep semiautomatic procedure. Briefly, two polyclonal rabbit antibodies for VEGF-C and VEGFR-3 (Santa Cruz Biotechnology Inc., Santa Cruz, CA, USA) at a dilution of 1:100 and 1:800, respectively, were used with an incubation time of $25 \mathrm{~min}$. For VEGF-D a polyclonal rabbit antibody (Zytomed, Berlin, Germany) was applied at a 1:100 dilution (25 min) after pretreatment with a steamer for antigen retrieval (Multi-Gourmet, Braun, no. 3216). After washing with phosphate-buffered saline (PBS, $0.01 \mathrm{~mol} / 1, \mathrm{pH} 7.3$ ) sections were exposed to biotinylated secondary antibody for $30 \mathrm{~min}$, followed by streptavidin-conjugated peroxidase for another $30 \mathrm{~min}$. Diaminobenzidine was applied as the final chromogen
Table I. Distribution of clinical and pathological variables in the reported series of ccRCC $(n=135)$ and pRCC $(n=31)$ specimens.

\begin{tabular}{|c|c|c|c|}
\hline & $\begin{array}{c}\mathrm{ccRCC} \\
\mathrm{n}(\%)\end{array}$ & $\begin{array}{l}\mathrm{pRCC} \\
\mathrm{n}(\%)\end{array}$ & $\mathrm{p}^{1}$ \\
\hline pT stage & & & 0.279 \\
\hline pT1 & $61(45.2)$ & $18(58.1)$ & \\
\hline pT2 & $11(8.1)$ & $1(3.2)$ & \\
\hline pT3 & 63 (46.7) & $12(38.7)$ & \\
\hline pN stage ${ }^{b}$ & & & 0.075 \\
\hline $\mathrm{pNx} / 0$ & $104(81.9)$ & $19(65.5)$ & \\
\hline $\mathrm{pN}^{+}$ & $23(18.1)$ & $10(34.5)$ & \\
\hline $\mathrm{cM} \mathrm{stage}^{\mathrm{c}}$ & & & 0.313 \\
\hline $\mathrm{cM} 0$ & $102(80.3)$ & $20(71.4)$ & \\
\hline $\mathrm{cM} 1$ & 25 (19.7) & 8 (28.6) & \\
\hline Grade $^{\mathrm{d}}$ & & & 0.010 \\
\hline G1 & $12(9.0)$ & $8(25.8)$ & \\
\hline G2 & 99 (73.9) & $15(48.4)$ & \\
\hline G3 & $23(17.2)$ & $8(25.8)$ & \\
\hline
\end{tabular}

anformation was available in $135(100 \%)$ of ccRCC and $31(100 \%)$ of pRCC. 'Information was available in 127 (94.1\%) of ccRCC and $29(93.5 \%)$ of pRCC. ' Information was available in $127(94.1 \%)$ of ccRCC and 28 (90.3\%) of pRCC. 'Information was available in 134 (99.3\%) of ccRCC and 31 (100\%) of pRCC. ${ }^{1} \mathrm{P}$-values from Pearson's $\chi^{2}$ and Fisher's exact test.

and nuclei were counterstained with haematoxylin to facilitate microscopic assessment. For the negative control, the primary antibody was replaced with PBS. No significant immunohistochemical reactions occurred in the control sections.

Quantification. A semiquantitative analysis of staining results from 332 tissue array cores was performed by two independent investigators blinded to the clinical and pathological data for the corresponding cases. For evaluation of the VEGF-C, VEGF-D and VEGFR-3 expression (all showing a cytoplasmatic and/or nuclear immunostaining pattern), the intensity of immunostaining was scored semiquantitatively on a four-tiered scale (negative, 0 ; weak, $1+$; moderate, $2+$ and strong, $3+)$. Samples with a moderate $(2+)$ or strong $(3+)$ immunostaining intensity were defined as having an increased expression of this marker and thus as 'positive', respectively (10) (Fig. 1). The final expression value for each tumor was the average round-up result of four single values (two representative regions for each tumor, two investigators).

Data analysis. For statistical analysis, SPSS for Windows (Version 15.0) was used. All clinical and pathological parameters were tested for their relationships with staining results by means of cross tables by applying Pearson's $\chi^{2}$ and Fisher's exact test. The Kaplan-Meier method was used for survival analysis and the log-rank test to compare the curves of two or 
Table II. Distribution of the positive expression results for VEGF-C, VEGF-D and VEGFR-3 in ccRCC and pRCC.

\begin{tabular}{lccccc}
\hline & VEGF-C positive (\%) & $\mathrm{p}^{1}$ & VEGF-D positive (\%) & $\mathrm{p}^{1}$ & VEGFR-3 positive (\%) \\
\hline ccRCC & $15(11.1)$ & 0.002 & $30(22.2)$ & 0.039 & $59(43.7)$ \\
pRCC & $11(35.5)$ & & $13(41.9)$ & & $19(61.3)$ \\
\hline
\end{tabular}

${ }^{1} \mathrm{P}$-values from Pearson's $\chi^{2}$ and Fisher's exact test.

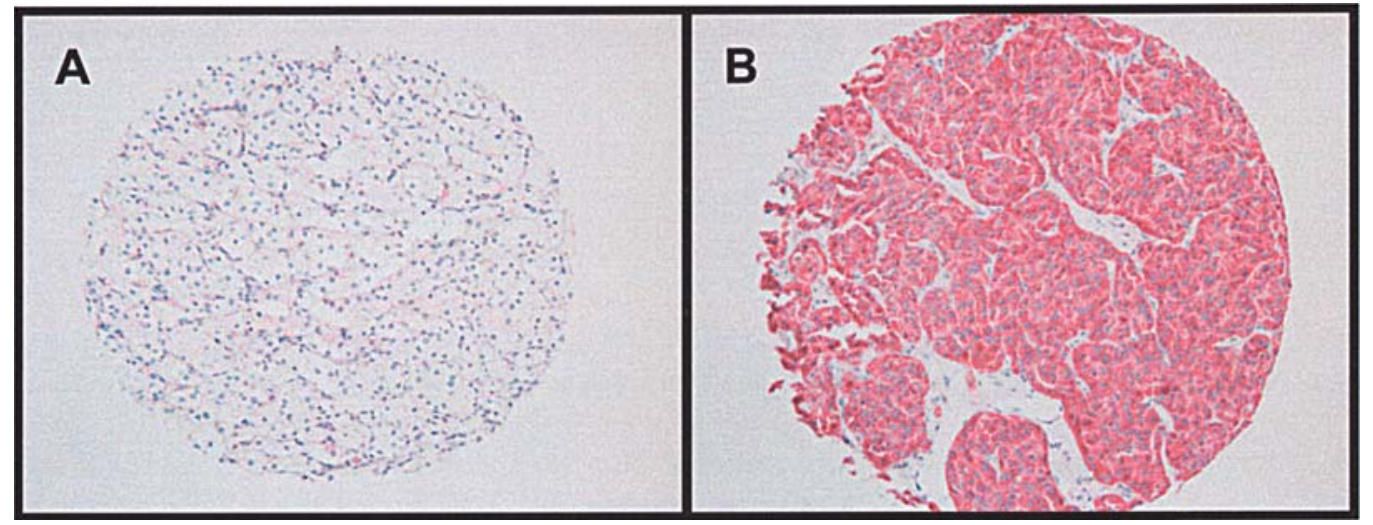

Figure 1. Representative examples of staining for VEGF-D. (A) Negative and (B) strong; images reduced from x20.

more groups. A p-value of $<0.05$ was considered statistically significant.

\section{Results}

Patient characteristics. One hundred and sixty-six patients treated with nephrectomy for localized, advanced or metastatic RCC at our institution between 1991 and 2004 were selected from our RCC database: 135 (81\%) of the patients had ccRCC and $31(19 \%)$ pRCC, respectively. Patients with other histological subtypes (e.g. chromophobe or sarcomatoid RCC) were excluded from our analysis. The median age of all patients at surgery was 63 years (range 25-84). None of the patients had received any prior therapy before surgery. As shown in Table I clinical and pathological parameters were well balanced between the two subgroups except for grading ( $\mathrm{p}=0.01)$. $\mathrm{pRCC}$ were slightly more often node-positive compared to ccRCC. However, this difference did not reach statistical significance $(\mathrm{p}=0.075)$.

Immunohistochemistry of VEGF-C, VEGF-D and VEGFR-3 expression. The staining status from all 166 patients was available. The staining intensity of VEGF-C, VEGF-D and VEGFR-3 among different samples ranged from the complete absence of staining to strong staining. In the two subgroups VEGF-C expression was significantly $(\mathrm{p}<0.001)$ correlated with the expression of VEGF-D. No correlation was observed between the expression of VEGF-C or VEGF-D and VEGFR-3 ( $p>0.05$, respectively). Expression results for ccRCC and pRCC are shown in Table II. VEGF-C expression was positive in $15(11.1 \%)$ of $c \mathrm{RCC}$ and $11(35.5 \%)$ of $\mathrm{pRCC}(\mathrm{p}=0.002)$.
An increased VEGF-D expression was detected in 30 (22.2\%) of ccRCC and 13 (41.9\%) of pRCC ( $\mathrm{p}=0.039)$. There was no significant difference regarding the expression of VEGFR-3 between the two subgroups [59 (43.7\%) in ccRCC and 19 $(61.3 \%)$ in $\mathrm{pRCC}, \mathrm{p}=0.11]$.

Correlation with clinicopathological parameters. The expression of VEGF-C, VEGF-D and VEGFR-3 was not associated with TNM stage or grading in either the entire cohort or in the two subgroups. The corresponding results for the entire group are shown in Table III. The median follow-up time was 28 months (range 4-120). The median overall and disease-free survival has not been achieved in the two subgroups at the time of analysis. The mean overall and disease-free survival in the entire cohort were 101 months [95\% confidence interval (CI): 93-108] and 80 months (95\% CI: 71-89), respectively. There was no association between survival (overall and disease-free) and the expression of the examined lymphangiogenic parameters (VEGF-C, VEGF-D and VEGFR-3) in either the subgroup or in the entire group.

\section{Discussion}

The molecular processes involved in lymphatic invasion and lymph node metastasis remain poorly understood. However, exciting discoveries have been made in the field of lymphangiogenesis in recent years. The identification of vascular endothelial growth factor ligands and cognate receptors involved in lymphangiogenesis, an understanding of the embryology of the mammalian lymphatic system, the recent isolation of pure populations of lymphatic endothelial cells, 
Table III. Correlation of the positive VEGF-C, VEGF-D and VEGFR-3 expression with clinical and pathological parameters.

\begin{tabular}{|c|c|c|c|c|c|c|}
\hline & $\begin{array}{l}\text { VEGF-C staining } \\
\text { n positive/total (\%) }\end{array}$ & $\mathrm{p}^{1}$ & $\begin{array}{c}\text { VEGF-D staining } \\
\text { n positive/total (\%) }\end{array}$ & $\mathrm{p}^{1}$ & $\begin{array}{l}\text { VEGFR-3 staining } \\
\text { n positive/total (\%) }\end{array}$ & $\mathrm{p}^{1}$ \\
\hline pT stage ${ }^{a}$ & & 0.502 & & 0.424 & & 0.467 \\
\hline pT1 & $13 / 79(16.5)$ & & $20 / 79(25.3)$ & & $41 / 79(51.9)$ & \\
\hline pT2 & $3 / 12(25.0)$ & & $5 / 12(41.7)$ & & $5 / 12(41.7)$ & \\
\hline pT3 & $10 / 75(13.3)$ & & $18 / 75(24.0)$ & & $32 / 75(42.7)$ & \\
\hline pN stage ${ }^{b}$ & & 1.000 & & 0.656 & & 0.557 \\
\hline $\mathrm{pNx} / 0$ & $21 / 123(17.1)$ & & $31 / 123(25.2)$ & & $55 / 123(44.7)$ & \\
\hline $\mathrm{pN}^{+}$ & $5 / 33(15.2)$ & & $10 / 33(30.3)$ & & $17 / 33(51.5)$ & \\
\hline cM stage $\mathrm{c}^{\mathrm{c}}$ & & 0.796 & & 0.657 & & 1.000 \\
\hline $\mathrm{cM} 0$ & 20/122 (16.4) & & $31 / 122(25.4)$ & & $57 / 122(46.7)$ & \\
\hline cM1 & 6/33 (18.2) & & $10 / 33(30.3)$ & & $15 / 33(45.5)$ & \\
\hline Grading $^{\mathrm{d}}$ & & 0.945 & & 0.926 & & 0.164 \\
\hline G1 & $3 / 20(15.0)$ & & $5 / 20(25.0)$ & & $13 / 20(65.0)$ & \\
\hline G2 & 19/114 (16.7) & & $29 / 114(25.4)$ & & $52 / 114(45.6)$ & \\
\hline G3 & $4 / 31(12.9)$ & & 9/31 (29.0) & & $12 / 31(38.7)$ & \\
\hline
\end{tabular}

${ }^{a}$ Information was available in $166(100 \%)$ cases. ${ }^{b}$ Information was available in $156(94.0 \%)$ cases. 'Information was available in 155 (93.4\%) cases. ${ }^{\mathrm{d} I n f o r m a t i o n}$ was available in $165(99.4 \%)$ cases. ${ }^{1} \mathrm{P}$-values from Pearson's $\chi^{2}$ and Fisher's exact test.

the investigation of lymphatic metastases in animal models, and the identification of markers that discriminate lymphatics from blood vessels at immunohistochemistry are current advances in understanding lymphangiogenesis (11). Moreover, an extensive range of animal models and clinicopathological studies suggest that lymphangiogenesis may play a key role in cancer progression (12). Therefore, lymphangiogenesis is probably one of the most interesting fields in cancer research at the moment.

The best validated molecular signalling system for tumor lymphangiogenesis involves the secreted proteins VEGF-C and VEGF-D that induce growth of the lymphatic vessels via activation of VEGFR-3 which is localised on the surface of lymphatic endothelial cells. A range of experimental studies in animal models (reviewed in ref. 13) has demonstrated that the VEGF-C/VEGF-D/VEGFR-3-axis can promote tumor lymphangiogenesis and the metastatic spread of tumor cells to the lymphatic system by inducing lymphatic endothelial cell growth, migration and survival (14). Clinicopathological correlations have shown that in various human cancers there is a strong correlation between this axis and lymph node metastasis $(12,13)$. Recent studies identified VEGF-D/ VEGFR-3 and VEGF-C as independent prognostic factors for poor prognosis in gastric adenocarcinoma (15) and cholangiocarcinoma (16), respectively. In addition, numerous reagents that may be used to block this pathway already exist, including soluble VEGFR-3 protein constructs, neutralising monoclonal antibodies to VEGFR-3 and VEGF-D, and small molecule inhibitors of the VEGFR-3 kinase, making the VEGF-C/VEGF-D/VEGFR-3-axis an attractive target for anti-lymphangiogenic therapy in cancer treatment (9).

Data focusing on lymphangiogenesis in RCC are scant. However, with regard to the VEGF-C/VEGF-D/VEGFR-3-axis some data are available. Gunningham et al reported that VEGF-C and VEGFR-3 are expressed in normal kidney tissue and renal cell carcinoma samples by measuring mRNA levels in a small series. Surprisingly, although an increase was observed, the VEGF-C and VEGFR-3 expression, was not significantly higher in the tumor compared with normal kidney tissue. Furthermore, VEGF-C and VEGFR-3 expression was not different between the most common subtypes of ccRCC and pRCC and there was no correlation with clinicopathological parameters such as tumor grade or size (17). Bando et al were able to stain VEGFR-3-positive vessels in renal cell carcinoma using a mouse monoclonal antibody. As expected the intratumoral VEGFR-3-positive vessel density was significantly correlated with the measured protein levels of VEGFR-3 by ELISA and the concentration of VEGFR-3 was significantly correlated with its ligand VEGF-C (18). Lin et al observed Caki-2 (RCC cell line) tumor-bearing animals that continuously expressed a soluble VEGFR-3 decoy receptor following virus-mediated gene transfer. In summary, they found a significant reduction of lymphatic vessels within the tumors and a $70 \%$ reduction of tumor cells in the lymph nodes compared to wild-type animals (19). Furthermore, they measured serum levels of the VEGFR-3 decoy receptor and demonstrated that inhibition of the lymph node metastases was dose-dependent. Thus, this study shows that the VEGF-C/ VEGF-D/VEGFR-3 signalling pathway appears to be a target for inhibiting lymphatic tumor spread in RCC.

In our study we analysed the expression of VEGF-C, VEGF-D and VEGFR-3 in a large number of RCC tumor samples using a tissue microarray and focusing on the two most common subtypes of ccRCC and pRCC, which differ substantially in a number of genetic, biological, pathological and clinical features (20). We demonstrated the expression of 
these factors in ccRCC and pRCC tumor specimens. In the two subgroups there was a significant correlation between the expression of VEGF-C and VEGF-D $(p<0.001)$ indicating that the secretion of these ligands may be regulated by the same mechanisms. Unlike Gunningham et al (17) we demonstrated that VEGF-C and VEGF-D expression was significantly higher in pRCC compared to ccRCC. However, although pRCC were more often node-positive than ccRCC ( 34.5 and $18.1 \%$, respectively, $\mathrm{p}=0.075$, Table I), no correlation was observed between the expression of VEGF-C or VEGF-D and the lymph node status (Table III). Moreover, in line with the reported literature we did not observe any correlation, either in the entire cohort or in the subgroups, between the expression of either VEGF-C or VEGF-D or VEGFR-3 and any clinicopathological parameter or survival data (overall and disease-free).

The expression of VEGFR-3 was not correlated with its ligands VEGF-C and VEGF-D. This is in contrast to the above-mentioned literature $(17,18)$, where a significant relationship between VEGFR-3 and VEGF-C levels (mRNA and protein levels) has been reported, but in agreement with studies in other tumor types $(12,15)$, where VEGFR-3 was not correlated with its ligands. This finding may indicate that in RCC the physiological effect of VEGF-C and VEGF-D is less to promote lymphangiogenesis by activating VEGFR-3 but more to signal via other receptors, e.g. VEGFR-2, since it is well known that the proteolytically activated forms of human VEGF-C and VEGF-D can also activate VEGFR-2, thereby promoting angiogenesis.

Our results show that the VEGF-C/VEGF-D/VEGFR-3axis is expressed in RCC with VEGF-C and VEGF-D being significantly up-regulated in pRCC compared to ccRCC. VEGF-C and VEGF-D expression was correlated with each other but not with the expression of its receptor VEGFR-3 and, in line with other reports, no correlation with clinicopathological parameters was shown in this study. Although evidence exists that the VEGF-C/VEGF-D/VEGFR-3-axis promotes lymphatic tumor spread in RCC (19), the exact link between the expression of this axis, tumor-induced lymphangiogenesis and clinical impact remains unclear. Therefore, further studies are necessary to determine the role of lymphangiogenesis and the VEGF-C/VEGF-D/VEGFR-3-axis in RCC. Future investigations should take the first international consensus on the methodology of lymphangiogenesis quantification in solid human tumors (21) into account and, as occurred in our study, strictly distinguish between different RCC subtypes. Then, it seems possible that this axis can play a role as a prognostic marker or possibly as a molecular target in different RCC subtypes.

\section{References}

1. Chow WH, Devesa SS, Warren JL and Fraumeni JF Jr: Rising incidence of renal cell cancer in the United States. JAMA 281: 1628-1631, 1999

2. Pantuck AJ, Zisman A and Belldegrun AS: The changing natural history of renal cell carcinoma. J Urol 166: 1611-1623, 2001.
3. Motzer RJ, Hutson TE, Tomczak P, Michaelson MD, Bukowski RM, Rixe O, Oudard S, et al: Sunitinib versus interferon alfa in metastatic renal-cell carcinoma. N Engl J Med 356: 115-124, 2007

4. Escudier B, Eisen T, Stadler WM, Szczylik C, Oudard S, Siebels M, Negrier S, et al: Sorafenib in advanced clear-cell renal-cell carcinoma. N Engl J Med 356: 125-134, 2007.

5. Hudes G, Carducci M, Tomczak P, Dutcher J, Figlin R, Kapoor A, Staroslawska E, et al: Temsirolimus, interferon alfa, or both for advanced renal-cell carcinoma. N Engl J Med 356: 2271-2281, 2007.

6. Escudier B, Pluzanska A, Koralewski P, Ravaud A, Bracarda S, Szczylik C, Chevreau C, et al: Bevacizumab plus interferon alfa-2a for treatment of metastatic renal cell carcinoma: a randomised, double-blind phase III trial. Lancet 370: 2103-2111, 2007.

7. Karakiewicz PI, Trinh QD, Bhojani N, Bensalah K, Salomon L, de la Taille A, Tostain J, et al: Renal cell carcinoma with nodal metastases in the absence of distant metastatic disease: prognostic indicators of disease-specific survival. Eur Urol 51: 1616-1624, 2007.

8. Canfield SE, Kamat AM, Sanchez-Ortiz RF, Detry M, Swanson DA and Wood CG: Renal cell carcinoma with nodal metastases in the absence of distant metastatic disease (clinical stage TxN1-2M0): the impact of aggressive surgical resection on patient outcome. $\mathrm{J}$ Urol 175: 864-869, 2006.

9. Achen MG, Mann GB and Stacker SA: Targeting lymphangiogenesis to prevent tumour metastasis. Br J Cancer 94: 1355-1360, 2006.

10. Wülfing P, Kersting C, Tio J, Fischer RJ, Wülfing C, Poremba C, Diallo R, et al: Endothelin-1-, endothelin-A-, and endothelin-Breceptor expression is correlated with vascular endothelial growth factor expression and angiogenesis in breast cancer. Clin Cancer Res 10: 2393-2400, 2004.

11. Sundar SS and Ganesan TS: Role of lymphangiogenesis in cancer. J Clin Oncol 25: 4298-4307, 2007.

12. Shayan R, Achen MG and Stacker SA: Lymphatic vessels in cancer metastasis: bridging the gaps. Carcinogenesis 27: 1729-1738, 2006.

13. Stacker SA, Williams RA and Achen MG: Lymphangiogenic growth factors as markers of tumor metastasis. APMIS 112: 539-549, 2004.

14. Makinen T, Veikkola T, Mustjoki S, Karpanen T, Catimel B, Nice EC, Wise L, et al: Isolated lymphatic endothelial cells transduce growth, survival and migratory signals via the VEGF-C/D receptor VEGFR-3. EMBO J 20: 4762-4773, 2001.

15. Juttner S, Wissmann C, Jons T, Vieth M, Hertel J, Gretschel S, Schlag PM, et al: Vascular endothelial growth factor-D and its receptor VEGFR-3: two novel independent prognostic markers in gastric adenocarcinoma. J Clin Oncol 24: 228-240, 2006.

16. Aishima S, Nishihara Y, Iguchi T, Taguchi K, Taketomi A, Maehara Y and Tsuneyoshi M: Lymphatic spread is related to VEGF-C expression and D2-40-positive myofibroblasts in intrahepatic cholangiocarcinoma. Mod Pathol (In press).

17. Gunningham SP, Currie MJ, Han C, Turner K, Scott PA, Robinson BA, Harris AL, et al: Vascular endothelial growth factor-B and vascular endothelial growth factor-C expression in renal cell carcinomas: regulation by the von Hippel-Lindau gene and hypoxia. Cancer Res 61: 3206-3211, 2001.

18. Bando H, Brokelmann M, Toi M, Alitalo K, Sleeman JP, Sipos B, Grone HJ, et al: Immunodetection and quantification of vascular endothelial growth factor receptor-3 in human malignant tumor tissues. Int J Cancer 111: 184-191, 2004.

19. Lin J, Lalani AS, Harding TC, Gonzalez M, Wu WW, Luan B, Tu GH, et al: Inhibition of lymphogenous metastasis using adenoassociated virus-mediated gene transfer of a soluble VEGFR-3 decoy receptor. Cancer Res 65: 6901-6909, 2005.

20. Kovacs G, Akhtar M, Beckwith BJ, Bugert P, Cooper CS, Delahunt B, Eble JN, et al: The Heidelberg classification of renal cell tumours. J Pathol 183: 131-133, 1997.

21. Van der Auwera I, Cao Y, Tille JC, Pepper MS, Jackson DG, Fox SB, Harris AL, et al: First international consensus on the methodology of lymphangiogenesis quantification in solid human tumours. Br J Cancer 95: 1611-1625, 2006. 